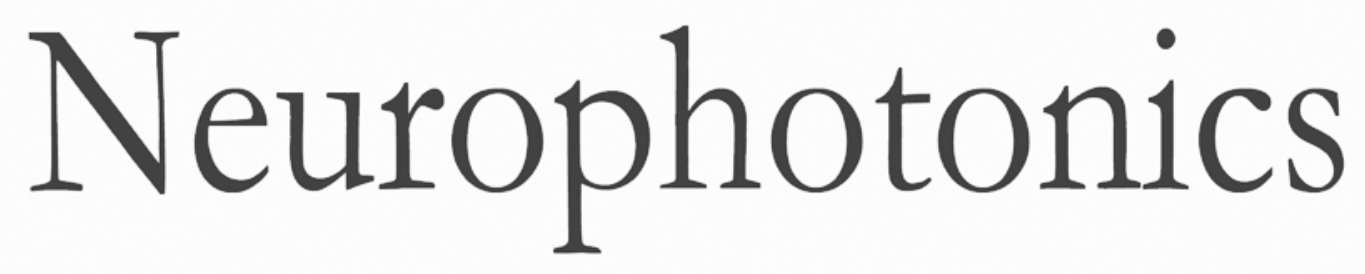

Neurophotonics.SPIEDigitalLibrary.org

\title{
Evolution of optogenetic microdevices
}

Rajas P. Kale

Abbas Z. Kouzani

Ken Walder

Michael Berk

Susannah J. Tye

\section{SPIE.}




\title{
Evolution of optogenetic microdevices
}

\author{
Rajas P. Kale, ${ }^{a, b}$ Abbas Z. Kouzani, ${ }^{a, \star}$ Ken Walder, ${ }^{c}$ Michael Berk, ${ }^{\text {d,e,f }}$ and Susannah J. Tye ${ }^{b}$ \\ aDeakin University School of Engineering, Faculty of Science, Engineering, and Built Environment, 75 Pigdons Road, \\ Waurn Ponds, Victoria 3216, Australia

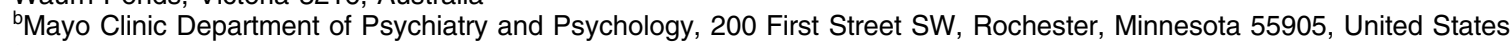 \\ ${ }^{\circ}$ Deakin University School of Medicine, 75 Pigdons Road, Waurn Ponds, Victoria 3216, Australia \\ 'Deakin University, IMPACT Strategic Research Centre, Faulty of Health, School of Medicine, Barwon Health, Geelong, Victoria, Australia \\ eOrygen, National Centre of Excellence in Youth Mental Health, Department of Psychiatry, 35 Poplar Road, Parkville, Victoria 3052, Australia \\ fUniversity of Melbourne, Florey Institute of Neuroscience and Mental Health, 30 Royal Parade, Parkville Victoria 3052, Australia
}

\begin{abstract}
Implementation of optogenetic techniques is a recent addition to the neuroscientists' preclinical research arsenal, helping to expose the intricate connectivity of the brain and allowing for on-demand direct modulation of specific neural pathways. Developing an optogenetic system requires thorough investigation of the optogenetic technique and of previously fabricated devices, which this review accommodates. Many experiments utilize bench-top systems that are bulky, expensive, and necessitate tethering to the animal. However, these bench-top systems can make use of power-demanding technologies, such as concurrent electrical recording. Newer portable microdevices and implantable systems carried by freely moving animals are being fabricated that take advantage of wireless energy harvesting to power a system and allow for natural movements that are vital for behavioral testing and analysis. An investigation of the evolution of tethered, portable, and implantable optogenetic microdevices is presented, and an analysis of benefits and detriments of each system, including optical power output, device dimensions, electrode width, and weight is given. Opsins, light sources, and optical fiber coupling are also discussed to optimize device parameters and maximize efficiency from the light source to the fiber, respectively. These attributes are important considerations when designing and developing improved optogenetic microdevices. @ 2015 Society of Photo-Optical Instrumentation Engineers (SPIE) [DOI: 10.1117/1 .NPh.2.3.031206]
\end{abstract}

Keywords: optogenetics; portable microdevices; fiber coupling; tethering; behavioral tests; psychiatry; neurology.

Paper 14086VSSR received Dec. 15, 2014; accepted for publication May 27, 2015; published online Jun. 25, 2015.

\section{Introduction}

The introduction of targeted photostimulation on opsin-expressing neuronal cells provides the potential to transform our insight into the neural circuitry associated with complex behaviors. In particular, research on treatment of psychiatric mood disorders is expected to benefit from optogenetic techniques. ${ }^{1-4}$ More broadly, neuroscience will benefit, and preclinical psychiatric research models stand to gain much through this approach. This may aid in our comprehension of how conscious behaviors are altered through pathway modulations that resemble disease states. ${ }^{5,6}$ Furthermore, conventional treatment options have limited efficacy and significant side-effects, and the development of novel therapies is dependent on advances in neurobiology to identify druggable targets. ${ }^{7,8}$ Therefore, in order to both understand the brain's complexity and determine safe treatment methods, it is necessary to advance behavioral research by modulating brain activity through the optogenetic method in appropriate animal models. However, caveats exist to conventional benchtop optogenetic systems in that they are bulky, expensive, inefficient, and require the animal to be tethered to receive photostimulation.

Tethering of the animal is one particular limitation of current optogenetic systems. Tethering increases experimental risks due to tangling of the optical fiber cord around the animal or through the experience of unintended rotational forces when connected.

*Address all correspondence to: Abbas Z. Kouzani, E-mail: abbas.kouzani@ deakin.edu.au
These issues confound natural behaviors that are essential for interpreting behavioral data. In general, behavioral tests should mimic previously validated studies rather than be unnecessarily modified. ${ }^{9}$ Having these established comparisons allows researchers to better correlate behaviors with the induced neural modulations. In the optogenetic technique, tethering the animal to the benchtop light source and control system substantially reduces the reproducibility of validated behavioral tests. One solution to this issue is to miniaturize the entire system such that photostimulation can be controlled and delivered through a wearable or implanted microdevice, ensuring minimal behavioral disturbances originate from the optogenetic system.

Despite the advantages for investigators to utilize a portable optogenetic system for neuromodulation research, the complexities in developing these microdevices necessitate advanced fabrication techniques and novel electronic components. Another difficulty arises when optimizing the coupling efficiency between the light source and the optical fiber to deliver adequate light power output while minimizing energy consumption. Therefore, many research groups continue to evolve tethered systems, focusing advances on unique optical electrodes and recording capabilities. As many research groups aim to develop their own device for their specific research protocols, taking an optogenetic microdevice from conception to production requires an extensive examination of both previously developed devices and cutting-edge technologies that can be implemented. To do

$2329-423 X / 2015 / \$ 25.00$ (C) 2015 SPIE 
this effectively, a current investigation of tethered, portable, and implantable devices is required for the analysis of the technological direction that these devices are taking, the studies they are facilitating, the advanced components each utilizes, as well as important parameters critical to each of their designs.

\section{Optogenetics}

\subsection{Opsins}

Optogenetics induces modulations in the activity of transfected neurons that express opsins on their membrane surface. When activated by light, these opsins function as channels that allow passage of specific ions to either depolarize or hyperpolarize the cell, affecting signal transmission. The result is the ability to control activation patterns of specific transfected cells through light delivery. ${ }^{10}$ Viral vectors are commonly used to transfect neurons in vivo. The virus is modified to only target certain cell types, for example, only those cells with Cre-positive expression. ${ }^{11}$ Once targeted, the virus splices the opsin-encoding DNA into the neuronal cell genome at the site of virus injection. Global opsin expression can alternatively be accomplished by genetically engineering transgenic animals. Each type of opsin has unique properties in terms of which ions it gates and what wavelengths activate it. Opsins commonly used for optogenetics research include halorhodopsin, channelrhodopsin, and archaerhodopsin (Table 1). Here we include the optimal activation wavelength for these opsins, as well as their effective power density for 50\% activation (EPD50). EPD50 measures photostimulation sensitivity of opsins independent of opsin expression levels, indicating how much optical output an optogenetic system needs to activate certain opsin types. With advances in genetic engineering, opsins can be developed to respond to a wider range of stimulation parameters, function through unique gating types, and have different sensitivities to light. Opsins have traditionally acted as channels to change ionic concentrations inside the cell. Opsins have also been engineered to activate second messenger systems, such as the OptoXR's Opto- $\alpha_{1} \mathrm{AR}$ ( $\mathrm{IP}_{3}$, DAG Recruiting GPCR) and Opto- $\beta_{2} \mathrm{AR}$ (cAMP Recruiter GPCR), which both activate at $473 \mathrm{~nm} .{ }^{12}$

\subsection{Light Sources}

Optimizing light sources and a delivery system is necessary to activate opsins at the region of interest, as optical penetration through brain tissue is relatively low from the cortical surface. Therefore, in order to modulate deeper brain regions, the light beam would need to be sufficiently strong at the site of stimulation. This is typically achieved through the use of optical fibers, but miniature light sources implanted into the brain can also be used. The light source must be powerful enough to penetrate enough tissue volume and stimulate the entire region of interest. Modeling light transmission through brain slices aids in determining how much light is required and can be calculated using the Kubelka-Munk equation for diffuse scattering media.

$$
T=\frac{1}{(S \times z)+1},
$$

where $T$ is the transmission fraction, $S$ is the scattering coefficient per unit thickness, and $z$ is the thickness of the sample. ${ }^{19}$ This simplified model demonstrates that transmission efficiency is inversely proportional to the scattering coefficient. Among the visible light spectrum ( 400 to $900 \mathrm{~nm}$ ), transmission loss from scattering is much greater than transmission loss from tissue absorption. ${ }^{20,21}$ This implies that the tissue scattering coefficient is an important indicator of light transmission through the brain tissue. For mice, the best fit scattering coefficient is $11.2 \mathrm{~mm}^{-1}$, while for rats, the best fit scattering coefficient is $10.3 \mathrm{~mm}^{-1} .15$

Aravanis et al. measured a $50 \%$ reduction in fiber-coupled optical power through $100 \mu \mathrm{m}$ of brain tissue penetration that increased to $90 \%$ after $1 \mathrm{~mm}$ of tissue depth using a $20 \mathrm{~mW}$ laser diode system. ${ }^{15}$ Laser systems penetrate through tissue with greater efficiency due to their low angle of incidence and high numerical aperture (see Sec. 2.3). The light exiting this $200 \mu \mathrm{m}$ multimodal fiber outputs is considerably high at $\sim 380 \mathrm{~mW} / \mathrm{mm}^{2}$ and spreads at an angle of $32 \mathrm{deg}$ with a numerical aperture of 0.37 . Neuronal activation is achievable at least $1.4 \mathrm{~mm}$ from the fiber tip, where optical power is at least $1 \mathrm{~mW} / \mathrm{mm}^{2}$ and covers a $1 \mathrm{~mm}$ diameter cross-section. With such high levels of tissue penetration and optical power, laserbased systems have proven useful in early optogenetic experiments. The first experiments demonstrating neuronal activation through a light source were performed in 1971 by Richard Fork using a $488 \mathrm{~nm}$ blue laser light at Bell laboratories. ${ }^{22}$ Their experiments required an extremely high level of irradiance $\left(140 \mathrm{~mW} / \mathrm{mm}^{2}\right)$, achievable through laser systems, to activate native abdominal ganglia of marine mollusks. Karl Deisseroth, who coined the term "optogenetics," first demonstrated millisecond-timescale activity control of Channelrhodopsin-2 (ChR-2) transfected mammalian cells using a $300 \mathrm{~W}$ xenon lamp in $2005 .{ }^{16}$ Since then, several light delivery devices have been introduced, including light-emitting diodes (LEDs), organic LEDs (OLEDs), liquid crystal displays (LCDs), halogen lights, and arc lamps. More recent systems have adopted LED systems over laser systems due to benefits in price, instrument size, beam stability,

Table 1 Characteristics and parameters of various opsins used in optogenetics.

\begin{tabular}{lcccc}
\hline Opsin type & Opsin subfamily & Gate type & Activation $\lambda(\mathrm{nm})$ & EPD50 (Ref. 13) \\
\hline Channelrhodopsin & ChR-2 & Cation channel & $460^{14-16}$ & $1.3 \mathrm{~mW} / \mathrm{mm}^{2}$ \\
Channelrhodopsin & VChR1 & Cation channel & $589^{17,18}$ & $\sim 0.5 \mathrm{~mW} / \mathrm{mm}^{2}$ \\
Halorhodopsin & eNpHR3.0 & Chloride pump & $589^{13}$ & $5.4 \mathrm{~mW} / \mathrm{mm}^{2}$ \\
Archaerhodopsin & Arch & Proton pump & $566^{12}$ & $\sim 7.5 \mathrm{~mW} / \mathrm{mm}^{2}$ \\
\hline
\end{tabular}

Note: EPD50, effective power density for $50 \%$ activation. 
and high frequency temporal precision. ${ }^{23}$ Despite the high power intensities achievable through laser-based systems, sufficient levels to activate ChR-2 proteins with $470 \mathrm{~nm}$ photostimulation are modest, with the minimum spiking irradiance being between 0.1 and $1 \mathrm{~mW} / \mathrm{mm}^{2}$ (or $10^{6}$ to $10^{7} \mathrm{~cd} / \mathrm{mm}^{2}$ ). ${ }^{24}$ At intensities $<0.1 \mathrm{~mW} / \mathrm{mm}^{2}$, ChR-2 response falls below $10 \%{ }^{25}$ High-intensity light sources, such as arc lamps ${ }^{16,26}$ and lasers, ${ }^{15,27,28,29}$ can achieve these optical outputs, as well as many LEDs; ${ }^{30-32}$ however, their function is limited to single-point illumination and whole-field illumination. OLEDs and LCDs, on the other hand, produce greater spatial resolution on two-dimensional arrays, but fall short on providing adequate irradiance for photoactivation (on the order of $1 \times 10^{-4} \mathrm{~mW} / \mathrm{mm}^{2}$, three orders of magnitude too low to activate ChR-2). ${ }^{33}$ Many newer optogenetic systems rely on LED light such as that developed by Clements et al. ${ }^{23}$ Their LED optogenetic system delivers adequate photostimulation to ChR-2 transfected neurons in mice to induce a freeze behavioral response, with peak light output from the device measuring at $29.6 \mathrm{~mW}$ through a $465 \mathrm{~nm}$ LED. With a total time lag of 328 microseconds from the trigger to $90 \%$ peak output, this LED-based device ensures the reliable temporal precision necessary for optogenetic stimulation parameters.

Photostimulation, especially with LEDs, has the benefit of producing relatively low stimulation artifacts during simultaneous neural recording. In contrast, when electrical recording devices are used with deep brain stimulation, large stimulation artifacts are present during pulse delivery. These recording systems include electroencephalogram recordings, ${ }^{34}$ evoked potential recording, ${ }^{35,36}$ local field potential recording, ${ }^{37}$ and single neuron recording. ${ }^{38}$ Each of these recording techniques needs to actively control artifact suppression through filtering and other methods to optimize signal-to-noise ratio during electrical stimulation. Three sources of stimulus artifacts can be identified at the recording electrode, including voltage gradients, common-mode conversion, and capacitive coupling. . $^{9,40}$ However, an analysis by Clements et al. ${ }^{23}$ of electrical recording during optogenetic stimulation revealed stimulation-induced noise that occurred at microvolt-scale detection levels compared to much larger millivolt-sized neuronal signals. ${ }^{23}$ Therefore, the perturbations of optogenetic stimulation artifacts are dwarfed by the electrical signals generated by the cells. These artifacts were recorded using a multielectrode array (platinum-iridium, pure iridium, tungsten); however, these low-frequency optical artifacts can also be picked up by electroencephalogram recordings or by local field potential electrodes. ${ }^{41}$ Low-frequency artifacts can be produced by a variety of processes ranging from temperature kinetics changing the electrode conductance to direct illumination of the metal electrode causing electron emissions (known as the photoelectric effect). ${ }^{42}$ These particular artifacts can be removed through high-pass filtering of the signal. $^{29}$

Delivering enough optical power to the site of stimulation is imperative and requires powerful light sources and efficient delivery systems. However, other parameters such as pulse width and frequency of light pulses are important characteristics of optogenetic stimulation parameters that need to be established for each study. Generally, a pulse width range of $100 \mu$ s to $10 \mathrm{~ms}$ and a pulse frequency up to $200 \mathrm{~Hz}$ covers the operating spectrum of optogenetic research. ${ }^{43}$ These parameters, however, are specific to the neuronal cell type, the expressed opsin, and the activation patterns specified for the experiment, and can vary greatly between experimental designs.
Determining the ideal parameters for an optogenetics study requires extensive research and experimentation.

\subsection{Fiber Coupling}

One of the challenges in building a novel optogenetic microdevice is deciding on an interface to optimally transmit light from the LED source to the neuronal tissue. Because power consumption is a major concern with both battery-based and energy-harvesting systems, the interface coupling should be as efficient as possible to reduce electrical demands. Some authors cited in this paper are satisfied with cortical surface stimulation to validate their device. However, most behavioral neuromodulation research focuses on deeper brain structures and requires a delivery method for the light. Optical fiber coupling is, therefore, an appropriate solution, as optical fiber materials efficiently transport light while being biocompatible and inert to organic tissue. Although some tissue damage does occur from optical fiber insertion, a relatively small "kill zone" is created when using small-diameter inert materials. ${ }^{44}$ Smaller-diameter optical fibers are more preferable than larger ones because they minimize neural necrosis along the insertion tract, as well as the resulting inflammatory response. ${ }^{45}$ However, smaller fibers transmit light less efficiently because of their more restrictive fiber cores. In rodents, fiber diameters of up to $300 \mu \mathrm{m}$ can be tolerated by mice, while up to $400 \mu \mathrm{m}$ can be tolerated by rats. ${ }^{46}$

Maximizing the coupling efficiency between the LED and the optical fiber is important but can be difficult due to the incredibly small optical fiber core compared with most source LEDs. Typical LEDs fall in the hundreds of micrometers to millimeter range, while micro-LEDs ( $\mu$ LEDs) are in the tens of micrometers range. ${ }^{33,47,48}$ Although the dimensions of most $\mu$ LEDs are comparable in size to the diameter of an optical fiber core, transmission efficiency is dependent on many factors including the LED's angle of incidence. A smaller core allows for less incident light to enter and accepts only smaller angles that propagate from the source. Therefore, multimodal optical fibers, which have much larger core diameters compared with single-mode fibers, offer better light transmission. A higher numerical aperture (NA) will also offer better coupling efficiency as it allows less light to escape through the fiber cladding once it has entered the core. The NA can be defined as

$\mathrm{NA}=\sqrt{n_{\text {core }}^{2}-n_{\text {clad }}^{2}}$,

where $n_{\text {core }}$ is the refractive index of the core, and $n_{\text {clad }}$ is the refractive index of the cladding around the core. The NA is, therefore, an important value to define the light-gathering capacity of the fiber. ${ }^{49}$ Using a lens also aids in focusing the light directly into the fiber; however, this increases the dimensions of the microdevice, as spacing is needed between the lens and the fiber equivalent to the focal length of the lens. Many costeffective lenses in the market have relatively large focal lengths $(\sim 3 \mathrm{~mm})$, while lenses made of more sophisticated materials have smaller focal lengths but can be costly. Lenses can greatly increase the coupling efficiency of the light to the fiber as shown by the following equation:

$\eta=\left[\frac{\mathrm{NA}_{(\text {fiber })}}{\mathrm{NA}_{(\text {lens })}}\right]^{2}$,

where $\eta$ is the coupling efficiency. This equation demonstrates that the coupling efficiency can be increased with a lens as long 
as the NA of the fiber is greater than the NA of the lens Typically, it is important to consider lenses with a refractive index of 1.9 or greater; otherwise, the peak coupling efficiency becomes equal to butt-coupling. ${ }^{50}$

Conventional methods for light delivery rely on acute optical fiber stimulation, where the optical fiber can be inserted and removed. The fiber is inserted only when the stimulation is required, for example, during behavioral testing. ${ }^{15}$ This design makes use of permanently fixed cannulas, ${ }^{15}$ such as those available from Doric Lenses. ${ }^{51}$ These cannulas are patched to minimize infection of exposed neural tissue, but can be opened whenever a fiber needs to be inserted for experimentation. The drawbacks from this method are apparent, in that continuous reinsertion of the fiber can increase the likelihood of breaking the fiber and clogging the cannula, ${ }^{46}$ as well as slightly changing the placement of the stimulation site and altering different neuronal activities during each fiber placement. ${ }^{50}$ In contrast, permanently fixing only the optical fiber into the brain without using a cannula leads to less tissue damage during chronic behavioral experiments due to the thinner implant. ${ }^{50,52}$ Many researchers use only a short fiber segment that is permanently implanted into the brain. ${ }^{53}$ When stimulation is needed, a fiber-to-fiber connector can couple this segment to a longer fiber that is connected to the light source. However, this connector can result in up to a $50 \%$ loss in transmission efficiency and, therefore, requires a powerful light source. Some limitations to this approach do exist, such as unintended escaping of LED light acting as a behavioral cue during stimulation. ${ }^{54,55}$

\section{Devices}

\subsection{Tethered Systems}

The optogenetic system by Clements et al. ${ }^{23}$ is a tethered device. This means that throughout light delivery, the animal must be connected by an optical fiber cord to a light source that is external to the animal's testing environment, as shown in Figs. 1(a) and 1(b). Tethered systems typically face a problem with tangling between the optical fiber cord and the animal, especially

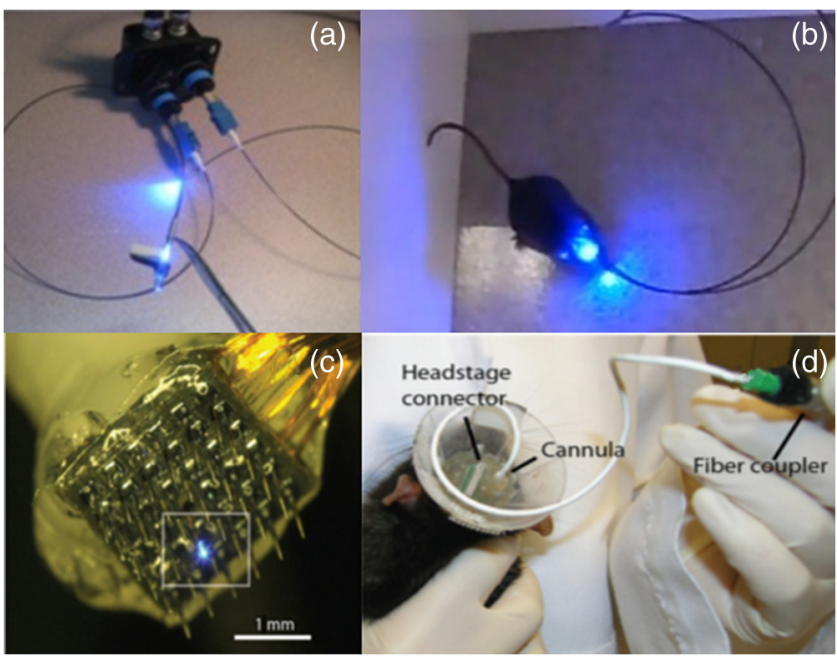

Fig. 1 (a) and (b) Optogenetic system by Clements et al. ${ }^{23}$ with a tethered animal. (c) and (d) A multielectrode and optrode array by Wang et al. ${ }^{58}$ capable of recording and stimulating a tethered animal. [Source: (a) and (b) reprinted from Clements et al. ${ }^{23}$ with permission from SPIE. (c) and (d) reproduced with permission from Wang et al., ${ }^{58}$ Copyright year 2012, IOP Publishing. All rights reserved.] if more than one animal is needed to study social behaviors. A rotary connector can be used to ensure free-moving behavior and uninterrupted optical connection within an open-topped behavioral apparatus, but they restrict experiments that require an enclosed home-cage top. ${ }^{56}$ Although a rotary connector seems to be an ideal solution for tethered experiments, it causes continuous pulling forces on the animal due to retraction of the cord, and frictional torques during animal rotations. Careful consideration of the animal species is important when using a rotary connector, as mice are able to withstand torques of up to $150 \mu \mathrm{N} \cdot \mathrm{m}$, while rats can exceed torque tolerations of $>300 \mu \mathrm{N} \cdot \mathrm{m} .^{57}$ Therefore, optimization of pulling forces by the rotary connector is necessary to keep the cord taunt without causing unnecessary cord retracting stress to the animal. Despite achieving rotary optimization, tethering can still alter behavior and disrupt the naturalistic testing environment being sought, potentially confounding behavioral experiments. Another issue with the use of rotary connectors is the transmission efficiency of the light source. Some commercially available rotaries yield relatively high transmission efficiencies, while some give much less (Prizmatix: 89\%, Doric Lenses: 85\%, ThorLabs: $65 \%$ ). Tethered systems are not limited by the electrical demands of their light source and can, therefore, deliver higher optical power. Compared with portable devices, which are restricted by their power supply and energy consumption, rotary connectors do benefit an experiment when tethering cannot be avoided.

Another function that can be conducted concurrently with photostimulation is neuronal recording. Recording the electrical signals of neurons produced by optogenetic stimulation gives insight into the robustness of the photostimulation response and subsequent spatial activity changes. Confirmation of continuous neuromodulation by the optogenetic system is achieved through these recording electrodes, providing both temporal and spatial information. Single recording electrode implants can measure the effectiveness of the optogenetic device and are, therefore, important tools to validate the device's functionality. Multielectrode arrays can measure the distribution of neuronal activation from the light beam as it disperses out of the fiber and into the tissue. This is because of the two-dimensional spatial recording abilities of multielectrode arrays. Previous applications of multielectrode arrays include neural network dynamics ${ }^{59}$ synaptic plasticity, ${ }^{60}$ visual perception, ${ }^{61}$ and pharmacotherapies. ${ }^{62}$ Because optical transmission decreases as the light passes through neural tissue [as shown by Eq. (1)], multiple recording electrodes can help determine how much area of activation is produced by the photostimulation. This is a useful tool to ensure that a specific brain region of interest can achieve the desired spatial coverage to ensure complete stimulation. Although each recording electrode on the array can be replaced with an electrical stimulator, conventional stimulation arrays are only able to stimulate, and not inhibit electrical activity.

Current technology allows for multielectrode arrays to be fitted with single optical fibers such as that by Wang et al. ${ }^{58}$ As shown in Figs. 1(c) and 1(d), a Pt-tipped $6 \times 6$ multielectrode array tapered to $1 \mathrm{~mm}$ length with $400 \mu \mathrm{m}$ spacing was used to provide verification that electrical activity was modulated locally in response to the light. Additionally, this system can be adapted with closed-loop control features to set stimulation triggers based on surrounding activity. These triggers ensure that activity is modulated only when certain neural criteria are met, helping to achieve the relevant behaviors at optimized 
times without overstimulating the brain. This is an important control feature in attenuating neurobiological diseases in animal models, such as epilepsy, because they can be turned on as soon as seizure-like waveforms are detected. ${ }^{63-65}$ However, one drawback that can arise with such multielectrode arrays is that multiple deep penetration points can cause brain injury during insertion, especially when trying to stimulate and record from deeper brain regions. Each recording electrode in the array can be hundreds of micrometers in diameter, and each causes neuronal damage as the array is driven into the brain. For this reason, most studies using multielectrode arrays restrict their measurements to cortical recording. The recording array of Wang et al., ${ }^{58}$ for example, only penetrated 0.5 to $1 \mathrm{~mm}$ into the brain. This kind of invasive surgery can trigger a prolonged inflammatory and immune response ${ }^{66}$ that could subsequently affect neural activity and confound behavior.

To avoid multiple penetration points during insertion of the optical fiber and recording electrodes, as well as to record from deeper brain regions, a solution is to combine the electrode and LED onto a biocompatible material as a single piece, commonly referred to as an optrode, and insert this into the desired region. This combined optrode can simultaneously stimulate and record from the same site and is a feature in the optogenetic system fabricated by Cao et al. ${ }^{67}$ The team incorporated the LED and electrode onto a single optrode for dual stimulation and recording as shown in Figs. 2(a) and 2(b). Their design minimizes power consumption by allowing full exposure of the LED to the stimulation site without losing efficiency through optical fiber coupling. This particular optrode is relatively wide, measuring $900 \mu \mathrm{m}$. In contrast, commercially available optical fibers for preclinical research are between 200 and $400 \mu \mathrm{m}$. The system of Cao et al. ${ }^{67}$ also uses a particularly large LED, measuring $1000 \mu \mathrm{m} \times 600 \mu \mathrm{m} \times 200 \mu \mathrm{m}$ (PicoLED, Rohm Semiconductor, Kyoto, Japan). However, technology is continuously advancing to produce smaller and more efficient LEDs that may be implemented in future designs. With advanced fabrication technologies and components, this device has potential to be reduced in size for less traumatic implantation. Early optrodes for tethered freely moving experiments were first developed by Anikeeva et al. ${ }^{68}$ to record medial prefrontal cortex response by local optical excitation and inhibition. Their group produced four $25-\mu \mathrm{m}$-diameter polymer-coated nickel-chromium alloy tetrode bundles that wound around a single $200 \mu \mathrm{m}$ optical fiber. The optrode was attached to a headstage to allow open-field movement while tethered to the light source.

In their experiment, Cao et al. ${ }^{67}$ bypassed the ChR-2 transfection process by procuring a transgenic Thy1-ChR2-YFP mouse line. While anesthetized and in stereotaxis, the mouse received blue light at $465 \mathrm{~nm}$ to the visual cortex created by an LED, which changed neuronal electrophysiology monitored by the recording optrode. Using transgenic animals can be advantageous in that the opsin molecules are persistently expressed throughout the brain. Unfortunately, obtaining such transgenic animals requires greater resources and time than utilizing the transfection process, as transgenic animals need to be genetically engineered and bred for several generations. Therefore, in most cases, recombinant viral vectors are used to administer the virus several weeks prior to the optrode insertion at the site of

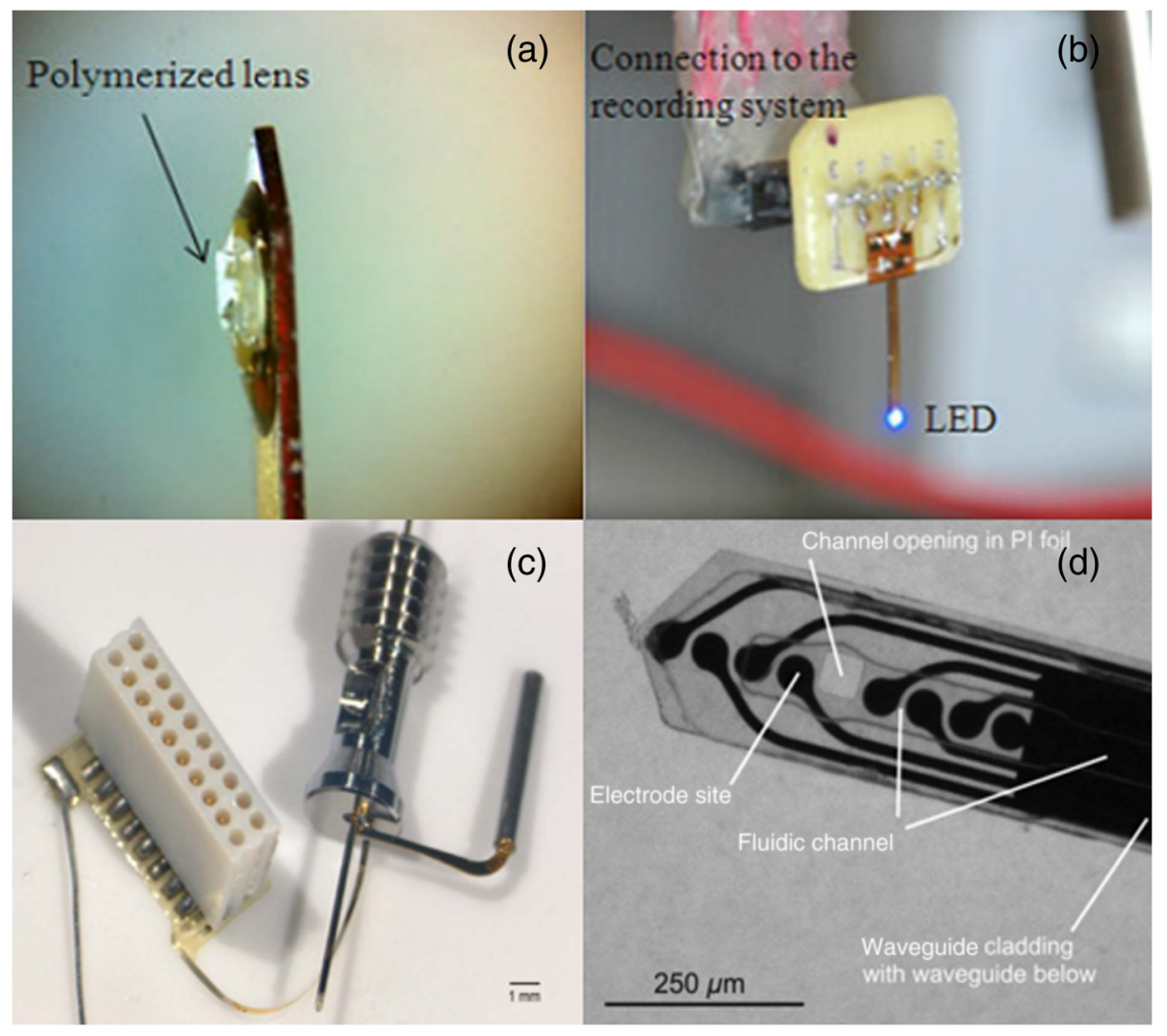

Fig. 2 (a) and (b) An optrode fabricated by Cao et al. ${ }^{67}$ for dual stimulation and recording from a single site. (c) and (d) An intricate recording/stimulating optrode by Rubehn et al. ${ }^{69}$ with a microfluidic channel capable of delivering a virus for neural transfection. [Source: (a) and (b) are reprinted from Cao et al. ${ }^{67}$ with permission from IEEE. (c) and (d) are reprinted from Rubehn et al.$^{69}$ with permission from Royal Society of Chemistry.] 
stimulation. The virus is typically administered by direct injection into the brain region of interest using a micro-syringe. The optrode is then implanted into the same spot after the virus is completely transfected. This double-surgery approach, however, adds surgical stress to the animal through anesthesia induction. Although unavoidable in most nontransgenic animals, the initial transfection surgery would have relatively little consequence in neurodegeneration because neuronal loss and the glial-inflammation response are considerably higher with persistent foreign body implantation than acute stab wounds followed by object removal. ${ }^{70}$ In general, glial response is biphasic with an early activation of microglia and astrocytes followed by a restriction of glial activity at the site closest to the implant (glial scarring). ${ }^{71}$ Nevertheless, maintaining opsin expression at the site of stimulation can be difficult after the optrode has been implanted. When retransfection is necessary for longer experiments, a continuous local viral transfection can be accomplished through the implementation of microfabrication techniques and microfluidics.

Advances in microelectromechanical systems fabrication techniques have allowed scientists to shrink down medical devices to the size of tiny chips. Lab-on-chip devices utilize microfluidic channels to transport fluids to specific targets in order to perform extensive laboratory-grade research in tiny settings. Rubehn et al. ${ }^{69}$ have incorporated this technology to deliver the soluble virus through microfluidic channels while also performing optical stimulation and electrical recording on one single microfluidic optrode, as shown in Figs. 2(c) and 2(d). Not only does this allow a single invasive surgery to accomplish both transfection and stimulation, it also enables microfluidic injection of the virus for on-demand, continuous transfection. This is because cellular expression of virally transfected opsins diminishes over time, therefore, experimental timelines need to be kept shorter or retransfection needs to take place. Once sufficient time has elapsed to ensure recombinant viral transfection, optical stimulation can begin to modulate neural activity and concurrent electrical recording can take place. With such precise fabrication processes, this fluidic delivery optrode hybrid has a thickness of $\sim 250 \mu \mathrm{m}$, about the same thickness as many optical fibers used. However, manufacturing such an optrode is not typically possible in most laboratories, as it needs a sophisticated fabrication process. Prior research in design, materials, and transport simulations must be realized before even the first prototype can be constructed, therefore, fabricating this device from scratch can be a laborious task even after all of the fabrication equipment and machinery has been procured. Despite all of these issues, the authors were able to successfully inject both
ChR-2 and NpHR at multiple depths when inserting this microfluidic-optrode hybrid. After six weeks of recovery, they were able to optically stimulate brain cells with both 473-nm light and 593-nm light with output powers of 21 and $14 \mathrm{~mW}$, respectively, while concurrently recording neuronal activity changes.

The current paper focuses on the advances in device components and functionality to illustrate the evolution of optogenetic microdevices from simple tethered systems to novel wireless devices. However, it is worth noting here that technologies have not progressed solely in electrical components. Optical fibers, for example, have also seen improvements recently, such as the tapered optical fiber developed by Pisanello et al. ${ }^{72}$ This fiber allows for dynamic illumination control along each taper, which acts as a window for light to escape from the fiber. Tapers are formed by locally removing the coating at each site where it is needed. This optical fiber technology allows multiple areas of the brain to be selectively targeted by photostimulation along the track of the optical fiber through the use of a single light source. However, sophisticated calculations and simulations are necessary to predictably deliver enough optical power through the desired tapers.

\subsection{Portable Microdevices}

Although the technologies described thus far (summarized in Table 2) represent significant serial improvements in research since the inception of optogenetic devices, a critical factor for device development is portability. Some of the previously described optogenetic systems were only able to stimulate anesthetized animals while they lay motionless under stereotaxic conditions, while other studies used tethered systems to allow some movement. Acute stereotactic optogenetics studies may be suitable for research on neural connectomics, but behavioral experiments require free-moving animals, with minimal deviations from the animals' natural settings to gather relevant data. Tethering the animal to the optogenetic system deviates from this natural setting as it restricts home-cage activity, spontaneous pain, wheel running, and freely moving social interactions. ${ }^{73}$ With emerging technologies allowing biomedical devices to become smaller than previously imagined, researchers have designed devices that are small enough to be implanted subcutaneously, ${ }^{57,74,75}$ some with only minor components exposed externally. These implantable devices allow the animal to perform measureable behavioral tasks while freely moving. Affixing headstage devices or optical fibers and cannulas to the animal requires the skull to be largely exposed during surgery. This exposure allows for adequate anchoring of cranial

Table 2 Parameters of tethered systems. Blank fields indicate no information given.

\begin{tabular}{lcccc}
\hline Parameter & Ref. 23 & Ref. 58 & Ref. 67 & Ref. 69 \\
\hline LED pulse width & $500 \mu \mathrm{s}$ & $20 \mathrm{~ms}$ & 25 to $100 \mathrm{~ms}$ & $300 \mu \mathrm{s}$ \\
Pulse frequency & $1 \mathrm{HZ}$ & $8 \mathrm{~Hz}$ & $1 \mathrm{to} 20 \mathrm{~Hz}$ & $0.5 \mathrm{~Hz}$ \\
Wavelength & $465 \mathrm{~nm}$ & $473 \mathrm{~nm}$ & $465 \mathrm{~nm}$ & $474 / 593 \mathrm{~nm}$ \\
Light output & $29.6 \mathrm{~mW}$ & $1.8 \mathrm{~mW}$ & $25 \mathrm{mcd}$ & $21 \mathrm{~mW} / 14 \mathrm{~mW}$ \\
Electrode width & $16 \mu \mathrm{m}$ & $200 \mu \mathrm{m}$ & $900 \mu \mathrm{m}$ & $250 \mu \mathrm{m}$ \\
Power density & & $916 \mathrm{~mW} / \mathrm{mm}^{2}$ & $0.7 \mathrm{~mW} / \mathrm{mm}^{2}$ &
\end{tabular}


screws and coverage of dental cement to ensure a strong bond is formed, helping keep the fixtures attached to the skull. The large surgical site, use of bonding cement, and drilling of cranial screws increase the stress on the animal associated with surgery, and should be minimized. Smaller and implantable devices help minimize these issues; this is one advantage of miniaturizing portable devices. Just as important, small devices give the animal less material to grab on-to during self-grooming. A problem associated with animal termination from a study is the animal's ability to grab a device or electrode and forcibly detach it from their skull. This leads to damage to the device, a loss of resources and time spent on surgery and treatments, and increase in the risk of injury or death to the animal.

The ability to implant a microdevice with no bulky components can remedy animal mortality rates. By eliminating the battery and harvesting energy through alternative sources, newer and smaller devices can be fabricated that are easier to implement in preclinical research. A small chip measuring $<9 \mathrm{~mm}^{2}$, constituting the bulk of the device, is an important aspect of the device of Yeh et al., ${ }^{74}$ as seen in Fig. 3(a). This is achievable through electromagnetic (EM) midfield energy harvesting, which the device gathers from an exposed coil measuring only $3 \mathrm{~mm}^{2}$. This energy accumulates onto a capacitor to power the LED. The source of this propagating field comes from a circuit board transmitting $500 \mathrm{~mW}$ of power, which is about the average power level of cell phones, to sustainably power the receiving coil even at distances of $15 \mathrm{~cm}$. This system propagates energy in the gigahertz spectrum within a range that is safe when absorbed by biological tissue. This desired frequency range can be sufficiently estimated as a time scale of the amplitude variation of the EM field falling largely between upper-bound and lower-bound values. The upper-bound value is represented by the dielectric relaxation time constant of the tissue, and the lower-bound value is represented by the electric time constant. Finding an ideal frequency range for energy harvesting is vitally important for the animal's wellbeing. The animal's wellbeing is scrutinized by institutional research ethics committees, and impacts on animal welfare profoundly affect experimental outcomes. ${ }^{76}$ Reported values for muscle tissue electric time constant and dielectric relaxation time constant are $2.3 \mathrm{~ns}$ and $7.2 \mathrm{ps}$, respectively, giving a safe midfield frequency range between $690 \mathrm{MHz}$ and $2.2 \mathrm{GHz}$. The Federal Communications Commission mandates that the maximum permissible exposure to radio frequency $(\mathrm{RF})$ power for humans in controlled environments is $3.03 \mathrm{~mW} / \mathrm{cm}^{2}{ }^{77}$ Although this particular device was developed to stimulate cardiac muscle cells in mice, it could be modified for optogenetic stimulation of neural tissue either directly by cortical stimulation or through the use of a fiber-optic cord for modulations in deep regions.

Other wireless and battery-less devices that can take advantage of power transmission are available. This concept is becoming more critical in the medical device field because of the inherent problems associated with thermodynamic chemical reactions that occur in them, including having a slow recharging rate of several hours, limited charge/discharge cycles, and being a biohazard if toxic materials are leaked. ${ }^{79,80}$ Batteries that sustain sufficiently long stimulation periods are inherently large and heavy, thus battery-based devices can interfere with the movements of animals. Therefore, it is beneficial to fabricate smaller battery-less microdevices to power the LED, which can have greater potential in recreating the animal's natural environment during photostimulation. One solution is substituting battery charging with capacitor charging through energy transmission as previously described. However, charging a capacitor to deliver energy to the LED would require multiple stacked capacitors to meet the high energy demands of most medical devices. Whereas batteries have sufficiently high energy densities, their power densities are relatively low, while capacitors have much higher power densities but lack in energy density. ${ }^{81}$ In order to solve this issue, a middle-ground is necessary, and one solution lies in the use of supercapacitors. Supercapacitors can achieve power densities several orders of magnitude greater than batteries. ${ }^{82}$ Meng et al. ${ }^{81}$ describe the fabrication of such supercapacitors that are flexible, solid-state, and are only $1 \mathrm{~mm}^{2}$ in size, yet offer a high area of capacitance at $1.3 \mathrm{mF} / \mathrm{mm}^{2}$. They proposed combining the supercapacitor circuit with a boost converter in order to minimize the current load on the supercapacitor, which will then charge a regular capacitor that regulates the power to the load.

Using a supercapacitor for miniature optogenetic devices powered by RF has the potential to substantially advance current behavioral research, enabling investigators to run multiple

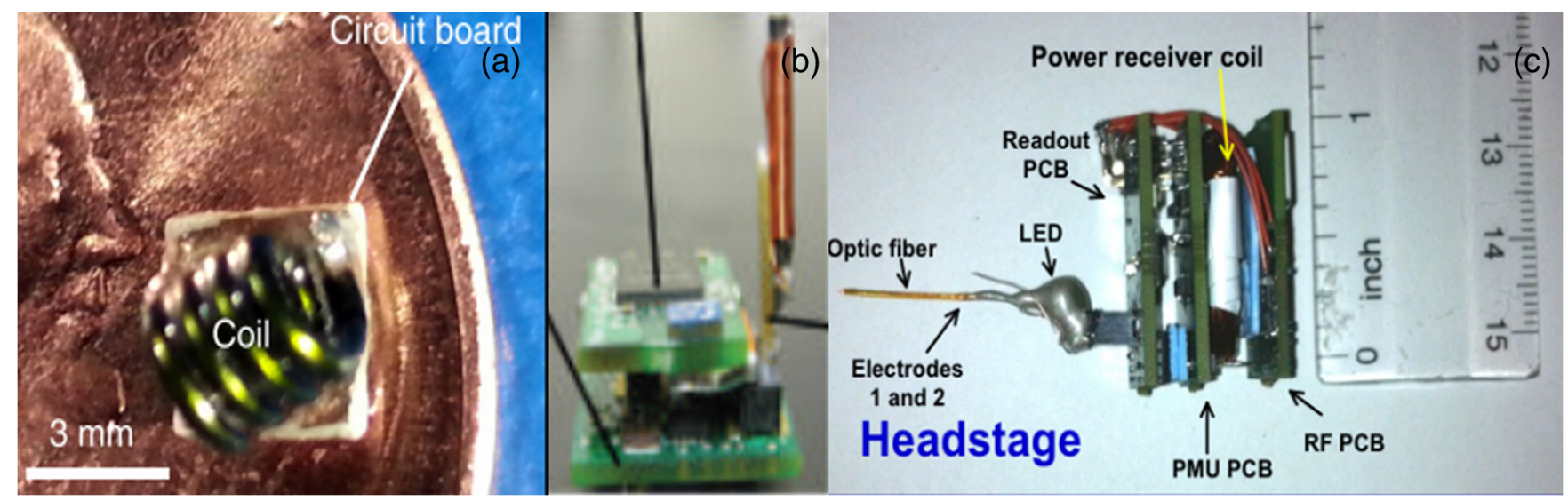

Fig. 3 (a) Transmission coil and circuit board described by Yeh et al. ${ }^{74}$ on a penny for size comparison. The tiny size of this system allows it to be implantable within the cranial skin of the animal. (b) The headstage and antenna developed by Wentz et al. ${ }^{43}$ (c) Headstage fabricated by Ameli et al. ${ }^{78}$ incorporating a recording electrode. [Source: (a) is reprinted with permission from Yeh et al. ${ }^{74}$ Copyright 2013, AIP Publishing LLC. (b) is reprinted with permission from Wentz et al. ${ }^{43}$ Copyright 2011, IOP Publishing. All rights reserved. (c) is reprinted from Ameli et al. ${ }^{78}$ with permission from IEEE.] 
animal studies in parallel. Devices with these capabilities are able to continuously transmit neurostimulation signals to a wearable or implanted optogenetic device while the animal is in its home cage. As shown in Fig. 3(b), Wentz et al. ${ }^{43}$ have developed such a device to demonstrate control of animal behaviors by stimulating adult Thy-ChR2 transgenic mice pyramidal cells of the motor cortex and analyzing subsequent rotational movements. Their device incorporated a low-strength oscillating magnetic field peaking at $300 \mathrm{~A} / \mathrm{m}$ to charge a supercapacitor, and power a $1 \times 1 \mathrm{~mm}^{2}$ LED with $2 \mathrm{~W}$ of power in steady state and $4.3 \mathrm{~W}$ in burst LED. Although their experimental setup was to stimulate cortical cells at the surface of the brain, their device can be adapted with fiber-optic cords to stimulate deeper regions. The whole device was fitted on a headstage that the animal wears and totals just $2 \mathrm{~g}$ in weight. Additionally, this device can be equipped with a $1 \mathrm{~g}$ wireless telemetry component, such as light detection or thermal data, to output data back to a computer. Throughout operation of the device, the animal is electrically insulated from the device components to ensure safety. The device is also monitored for heat generation from the LED and integrated circuit. The maximum temperature rise in steady-state operation was $0.6^{\circ} \mathrm{C}$, representing a worst case scenario and well within a safe range.

One additional method to further advance an optogenetic microdevice would be incorporating a recording electrode to wirelessly send information back to a computer for stimulation readouts. Ameli et al. ${ }^{78}$ have accomplished this task as shown in Fig. 3(c). Using a fiber-optic cord concurrently with electrodes coupled to a headstage device with a wireless signal rectifier, their device incorporates many of the evolutionary advances discussed throughout this review. Their device is relatively heavy at $7.4 \mathrm{~g}$, but implementing technological advances to the device, such as smaller components and custom integrated circuits, can solve this problem as discussed by the authors. Nevertheless, the device is small at $15 \times 25 \times 17 \mathrm{~mm}^{3}$. The transmitter can power the device up to $7 \mathrm{~cm}$ away and can transmit data back to a base-station computer $>2 \mathrm{~m}$ away. This makes it ideal for running studies with multiple animals in individual home cages receiving power through multiple transmission sources and sending the information back to a central computer. Although this device has not been tested in vivo, it demonstrates promise that future iterations can be developed that are smaller and robust.

Smaller components and devices are being progressively implemented in more recent portable optogenetic devices. Rossi et al. ${ }^{83}$ fitted their LED onto an optrode that can be inserted into the brain. This allows experiments by this device to be performed in deep brain regions without relying on optical fibers. Although these LEDs are considered relatively large compared to smaller $\mu$ LEDs, this device represents a marked improvement in portable devices compared to those previously discussed. As can be seen in Table 3, this newer device is smaller, lighter, and has a greater transmission range than other devices. It does, however, require a battery to recharge the device every $2 \mathrm{~h}$, and other limitations exist, such as a lack of recording features. One important advantage of this system is that all of the components are commercially available and the device can be constructed in any standard neuroscience lab. The ability for most laboratories to construct their own device becomes harder as the device dimensions are reduced, advanced components and functions are utilized, and more fabrication technologies are required.

\subsection{Implantable Devices}

Miniaturization of optogenetic devices is far from being completely realized, as newer classes of implantable optogenetic systems are being introduced that are several orders of magnitude smaller in scale compared to early systems. Implantable devices take advantage of $\mu$ LEDs that are inserted into the site of stimulation and are, therefore, less reliant on optical fibers to deliver light. Optical fibers are typically able to illuminate ventral structures from the fiber tip, whereas $\mu$ LEDs have the advantage of complete customizations, including the shape and size of the photostimulation. $\mu$ LEDs can come in a range of sizes $\left(625\right.$ to $\left.10,000 \mu \mathrm{m}^{2}\right)$, can be fitted on a two-dimensional array and spatially controlled for different illumination patterns, and can output light in different directions using reflective surfaces. ${ }^{73}$ Because of the low power demands of advanced semiconductor $\mu$ LEDs, power harvesting systems through radiofrequency scavenging make it possible for an optogenetic system to be at least partially implantable at the site of

Table 3 Transmission and device parameters of portable devices. Blank fields indicate no information given.

\begin{tabular}{|c|c|c|c|c|c|}
\hline Parameter & Ref. 74 & Ref. 43 & Ref. 78 & Ref. 83 & Refs. 73 and 84 \\
\hline LED wavelength & $590 / 630 \mathrm{~nm}$ & $470 \mathrm{~nm}$ & Various & $465.5 \mathrm{~nm}$ & Various \\
\hline LED size & Varies & $1 \times 1 \mathrm{~mm}$ & Varies & $240 \times 320 \times 140 \mu \mathrm{m}$ & $50 \times 50 \times 6.45 \mu \mathrm{m}$ \\
\hline Stimulation site & Tissue surface & Cortical surface & Anywhere via optical fiber & Anywhere via implant & Anywhere via implant \\
\hline Device dimensions & $9 \mathrm{~mm}^{2}$ & $<1 \mathrm{~cm}^{3}$ & $15 \times 25 \times 17 \mathrm{~mm}$ & $14 \times 17 \times 5 \mathrm{~mm}$ & $\sim 1 \mathrm{~cm}^{3}$ \\
\hline Weight & & $2 \mathrm{~g}$ & $7.4 \mathrm{~g}$ & $2.9 \mathrm{~g}$ & $\sim 2 \mathrm{~g}$ \\
\hline Receiver frequency & $1.6 \mathrm{GHz}$ & 2.4 to $2.485 \mathrm{GHz}$ & $868 \mathrm{MHz}$ & 2.4 to $2.5 \mathrm{GHz}$ & $910 \mathrm{MHz}$ \\
\hline Transmission range & $15 \mathrm{~cm}$ & Several $\mathrm{cm}$ & $<7 \mathrm{~cm}$ & $4 \mathrm{~m}$ & 1 to $2 \mathrm{~m}$ \\
\hline Data rate & & $1 \mathrm{Mbit} / \mathrm{s}$ & $320 \mathrm{Kbit} / \mathrm{s}$ & $250 \mathrm{Kbit} / \mathrm{s}$ & \\
\hline Power source & EM midfield & RF scavenging & Inductive power & Rechargeable battery & RF scavenging \\
\hline
\end{tabular}


stimulation. In partially implantable systems, only the power harvesting components are exposed outside of the skull to efficiently harness energy and ensure animal safety. An example of implantable devices that use wireless energy harvesting is the device developed by Kim et al. ${ }^{84}$ Through advanced microfabrication techniques as described in their protocol, ${ }^{73}$ their device demonstrates how multiple components can be integrated onto a miniature system. The system layers functional components onto an injectable microneedle to conveniently implant the optrode. The multitude of functions performed by this single optrode include electrophysiological measurements (Pt-contact pad, microelectrode), optical measurement (silicon $\mu$-IPD), optical stimulation ( $\mu$ LED array), and temperature sensing (serpentine Pt resistor). When dealing with functional components in close proximity to internal tissue, thermal management is imperative for the animal's safety and it is important to measure the temperature during device validation. Their device's $\mu$ LEDs produced $<0.10^{\circ} \mathrm{C}$ change in temperature, which is much lower than the $\sim 2^{\circ} \mathrm{C}$ temperature change produced by deep brain stimulation. ${ }^{85}$ At a distance of $1 \mathrm{~m}$ and a radio-frequency scavenger output of $4.08 \mathrm{~mW}$, the group was able to produce a $7 \mathrm{~mW} / \mathrm{mm}^{2}$ optical power output. The RF power that reaches the animal at this distance is between 0.15 and $0.77 \mathrm{~mW} / \mathrm{cm}^{2}$, much lower than the maximum permissible exposure limits for humans. These cellular-scale $\mu$ LEDs are only $6.45 \mu \mathrm{m}$ thick and $50 \times 50 \mu \mathrm{m}^{2}$ in area, which are more than a thousand times smaller than conventional LEDs. A collection of four $\mu$ LEDs on the single optrode was able to increase self-stimulated lever presses by mice associated with dopamine release in the ventral tegmental area.

Integration of $\mu$ LEDs onto an array is a useful tool to precisely stimulate brain regions with complex shapes or topographic neuronal cell types. In such cases, having the ability to target the region of interest without photostimulating other regions allows researchers to control neuromodulation experiments at the cellular scale. Grossman et al. ${ }^{33}$ implemented $4096 \mu$ LEDs, each only $20 \mu \mathrm{m}$ in diameter, to create a $4 \times 4 \mathrm{~mm}^{2}$ optogenetic array. Each $3 \times 3 \mathrm{~mm}^{2}$ light spot created by these $\mu$ LEDs produces up to $3 \mathrm{~mW} / \mathrm{mm}^{2}$ optical power. With lenses, this light spot can be demagnified by $10: 1$ to produce a $0.3 \times 0.3 \mathrm{~mm}^{2}$ light spot with $300 \mathrm{~mW} / \mathrm{mm}^{2}$. Each one of these $\mu$ LEDs can be individually switched on or off and contain different wavelength emitters for a broader range of opsin activation. This capability also allows $\mu$ LED arrays to activate certain clusters of neural populations within regions, maximizing activation precision. Research in sensory prosthetics and other studies involving topographic regions of interest can benefit from the cellular-scale precision offered by this device.

\section{Conclusion}

Advances in medical technology and fabrication techniques have enabled brain research and increased the scope of potential studies. New devices are constantly being developed and distributed for use by researchers. Tables 2 and 3 illustrate only a small portion of important considerations in developing optogenetics devices. Understanding the trends and advances is important for designing improved systems for up-to-date research methods. A device is within reach that would contain a modifiable LED source, recording electrodes, and a microfluidics viral delivery system all on a single optrode. The chip would be small enough to sit on top of the skull yet be implanted under the skin and incorporate a tiny rectenna that wirelessly powers the device, transmits information, and alters the parameters in real time. This way, animal behavior studies can be performed in large scale and in parallel. This will enable researchers to better understand the core neurobiology of diverse neurological and psychiatric disorders, and facilitate the rational development of treatments for wide-ranging neural syndromes, such as depression, epilepsy, Alzheimer's disease, and more.

\section{Acknowledgments}

Michael Berk is supported by an NHMRC Senior Principal Research Fellowship 1059660.

\section{References}

1. R. T. LaLumiere, "A new technique for controlling the brain: optogenetics and its potential for use in research and the clinic," Brain Stimul. 4(1), 1-6 (2011).

2. K. Deisseroth, "Controlling the brain with light," Sci. Am. 303(5), 48-55 (2010).

3. M. M. Sidor, "Psychiatry's age of enlightenment: optogenetics and the discovery of novel targets for the treatment of psychiatric disorders," J. Psychiatry Neurosci. 37(1), 4 (2012).

4. E. E. Steinberg and P. H. Janak, "Establishing causality for dopamine in neural function and behavior with optogenetics," Brain Res. 1511, 46-64 (2013)

5. A. V. Kravitz et al., "Regulation of Parkinsonian motor behaviours by optogenetic control of basal ganglia circuitry," Nature 466(7306), 622-626 (2010).

6. F. E. LeBeau, "Gamma oscillations and the cellular components of consciousness?," New Horiz. Neurosci. Conscious. 79, 29-37 (2010).

7. M. Berk and A. A. Nierenberg, "Three paths to drug discovery in psychiatry," Am. J. Psychiatry 172(5), 412-414 (2015).

8. M. Berk, "Pathways to new drug discovery in neuropsychiatry," $B M C$ Med. 10(1), 151 (2012).

9. A. Kalueff, M. Wheaton, and D. Murphy, "What's wrong with my mouse model?: advances and strategies in animal modeling of anxiety and depression," Behav. Brain Res. 179(1), 1-18 (2007).

10. K. Deisseroth, "Optogenetics," Nat. Methods 8(1), 26-29 (2011).

11. L. Madisen et al., "A toolbox of Cre-dependent optogenetic transgenic mice for light-induced activation and silencing," Nat. Neurosci. 15(5), 793-802 (2012).

12. B. Y. Chow et al., "High-performance genetically targetable optical neural silencing by light-driven proton pumps," Nature 463(7277), 98-102 (2010).

13. J. Mattis et al., "Principles for applying optogenetic tools derived from direct comparative analysis of microbial opsins," Nat. Methods 9(2), 159-172 (2012).

14. G. Nagel et al., "Channelrhodopsin-2, a directly light-gated cationselective membrane channel," Proc. Natl. Acad. Sci. 100(24), 1394013945 (2003)

15. A. M. Aravanis et al., "An optical neural interface: in vivo control of rodent motor cortex with integrated fiberoptic and optogenetic technology," J. Neural Eng. 4(3), S143 (2007).

16. E. S. Boyden et al., "Millisecond-timescale, genetically targeted optical control of neural activity," Nat. Neurosci. 8(9), 1263-1268 (2005).

17. F. Zhang et al., "Red-shifted optogenetic excitation: a tool for fast neural control derived from Volvox carteri," Nat. Neurosci. 11(6), 631-633 (2008).

18. J. K. Lanyi et al., "The primary structure of a halorhodopsin from Natronobacterium pharaonis. Structural, functional and evolutionary implications for bacterial rhodopsins and halorhodopsins," J. Biol. Chem. 265(3), 1253-1260 (1990).

19. T. Vo-Dinh, Biomedical Photonics Handbook: Biomedical Diagnostics, CRC Press (2014).

20. F. Bevilacqua et al., "In vivo local determination of tissue optical properties: applications to human brain," Appl. Opt. 38(22), 4939-4950 (1999).

21. A. Yaroslavsky et al., "Optical properties of selected native and coagulated human brain tissues in vitro in the visible and near infrared spectral range," Phys. Med. Biol. 47(12), 2059 (2002). 
22. R. L. Fork, "Laser stimulation of nerve cells in Aplysia," Science 171(3974), 907-908 (1971).

23. I. P. Clements et al., "Miniaturized LED sources for in vivo optogenetic experimentation," Proc. SPIE 8586, 85860X (2013).

24. P. Degenaar et al., "Optobionic vision: a new genetically enhanced light on retinal prosthesis," J. Neural Eng. 6(3), 035007 (2009).

25. N. Grossman et al., "Modeling study of the light stimulation of a neuron cell with channelrhodopsin-2 mutants," IEEE Trans. Biomed. Eng. 58(6), 1742-1751 (2011)

26. F. Zhang et al., "Channelrhodopsin-2 and optical control of excitable cells," Nat. Methods 3(10), 785-792 (2006).

27. V. Gradinaru et al., "Optical deconstruction of Parkinsonian neural circuitry," Science 324(5925), 354-359 (2009).

28. A. R. Adamantidis et al., "Neural substrates of awakening probed with optogenetic control of hypocretin neurons," Nature 450(7168), 420-424 (2007).

29. X. Han et al., "Millisecond-timescale optical control of neural dynamics in the nonhuman primate brain," Neuron 62(2), 191-198 (2009).

30. V. Gradinaru et al., "Targeting and readout strategies for fast optical neural control in vitro and in vivo," J. Neurosci. 27(52), 14231-14238 (2007).

31. J. G. Bernstein et al., "Prosthetic systems for therapeutic optical activation and silencing of genetically targeted neurons," Proc. SPIE 6854, 68540H (2008).

32. D. Huber et al., "Sparse optical microstimulation in barrel cortex drives learned behaviour in freely moving mice," Nature 451(7174), 61-64 (2008).

33. N. Grossman et al., "Multi-site optical excitation using ChR2 and micro-LED array," J. Neural Eng. 7(1), 016004 (2010).

34. N. Swann et al., "Deep brain stimulation of the subthalamic nucleus alters the cortical profile of response inhibition in the beta frequency band: a scalp EEG study in Parkinson's disease," J. Neurosci. 31(15), 5721-5729 (2011).

35. Y. Maegaki et al., "Somatosensory evoked high-frequency oscillations recorded directly from the human cerebral cortex," Clin. Neurophysiol. 111(11), 1916-1926 (2000).

36. D. S. Dinner et al., "EEG and evoked potential recording from the subthalamic nucleus for deep brain stimulation of intractable epilepsy," Clin. Neurophysiol. 113(9), 1391-1402 (2002).

37. L. Rossi et al., "An electronic device for artefact suppression in human local field potential recordings during deep brain stimulation," J. Neural Eng. 4(2), 96 (2007).

38. M. Filali et al., "Stimulation-induced inhibition of neuronal firing in human subthalamic nucleus," Exp. Brain Res. 156(3), 274-281 (2004)

39. K. C. McGill et al., "On the nature and elimination of stimulus artifact in nerve signals evoked and recorded using surface electrodes," IEEE Trans. Biomed. Eng. BME-29(2), 129-137 (1982).

40. A. Kent and W. Grill, "Recording evoked potentials during deep brain stimulation: development and validation of instrumentation to suppress the stimulus artefact," J. Neural Eng. 9(3), 036004 (2012).

41. J. G. Bernstein and E. S. Boyden, "Optogenetic tools for analyzing the neural circuits of behavior," Trends Cogn. Sci. 15(12), 592-600 (2011).

42. J. A. Cardin et al., "Targeted optogenetic stimulation and recording of neurons in vivo using cell-type-specific expression of Channelrhodopsin-2," Nat. Protoc. 5(2), 247-254 (2010).

43. C. T. Wentz et al., "A wirelessly powered and controlled device for optical neural control of freely-behaving animals," J. Neural Eng. 8(4), 046021 (2011)

44. S. S. Stensaas and L. Stensaas, "The reaction of the cerebral cortex to chronically implanted plastic needles," Acta Neuropathol. 35(3), 187203 (1975).

45. W. M. Grill, S. E. Norman, and R. V. Bellamkonda, "Implanted neural interfaces: biochallenges and engineered solutions," Апnи. Rev. Biomed. Eng. 11, 1-24 (2009).

46. M. E. Carter and L. de Lecea, "Optogenetic investigation of neural circuits in vivo," Trends Mol. Med. 17(4), 197-206 (2011).

47. B. McGovern et al., "A new individually addressable micro-LED array for photogenetic neural stimulation," IEEE Trans. Biomed. Circuits Syst. 4(6), 469-476 (2010).

48. Y. Iwai et al., "A simple head-mountable LED device for chronic stimulation of optogenetic molecules in freely moving mice," Neurosci. Res. 70(1), 124-127 (2011).
49. A. G. Rays and C. S.-M. Fibers, "Fiber optics," (1991).

50. S. J. Wetzel, "Coupling light emitting diodes to multimode optical fibers," 1993, http://preserve.lehigh.edu/cgi/viewcontent.cgi?article= 1165\&context=etd (29 May 2015)

51. www.doriclenses.com

52. G. D. Stuber et al., "Amygdala to nucleus accumbens excitatory transmission facilitates reward seeking," Nature 475(7356), 377 (2011).

53. F. Zhang et al., "Optogenetic interrogation of neural circuits: technology for probing mammalian brain structures," Nat. Protoc. 5(3), 439-456 (2010).

54. E. Martín-García et al., "New operant model of reinstatement of food-seeking behavior in mice," Psychopharmacol. 215(1), 49-70 (2011).

55. C. M. Olsen and D. G. Winder, "Operant sensation seeking engages similar neural substrates to operant drug seeking in C57 mice," Neuropsychopharmacology 34(7), 1685-1694 (2009).

56. B. B. Land et al., "Optogenetic inhibition of neurons by internal light production," Front. Behav. Neurosci. 8 (2014).

57. D. C. Klorig and D. W. Godwin, "A magnetic rotary optical fiber connector for optogenetic experiments in freely moving animals," J. Neurosci. Methods 227, 132-139 (2014).

58. J. Wang et al., "Integrated device for combined optical neuromodulation and electrical recording for chronic in vivo applications," J. Neural Eng. 9(1), 016001 (2012).

59. F. Hofmann and H. Bading, "Long term recordings with microelectrode arrays: studies of transcription-dependent neuronal plasticity and axonal regeneration," J. Physiol.-Paris 99(2), 125-132 (2006).

60. F. Lanté et al., "Low-frequency stimulation induces a new form of LTP, metabotropic glutamate (mGlu5) receptor-and PKA-dependent, in the CA1 area of the rat hippocampus," Hippocampus 16(4), 345-360 (2006)

61. J. Cang et al., "Development of precise maps in visual cortex requires patterned spontaneous activity in the retina," Neuron 48(5), 797-809 (2005)

62. S. J. Aton et al., "Vasoactive intestinal polypeptide mediates circadian rhythmicity and synchrony in mammalian clock neurons," Nat. Neurosci. 8(4), 476-483 (2005).

63. E. Krook-Magnuson et al., "On-demand optogenetic control of spontaneous seizures in temporal lobe epilepsy," Nat. Commun. 4, 1376 (2013).

64. J. T. Paz et al., "Closed-loop optogenetic control of thalamus as a tool for interrupting seizures after cortical injury," Nat. Neurosci. 16(1), 64-70 (2013).

65. C. Armstrong et al., "Closed-loop optogenetic intervention in mice," Nat. Protoc. 8(8), 1475-1493 (2013).

66. G. C. McConnell et al., "Implanted neural electrodes cause chronic, local inflammation that is correlated with local neurodegeneration," J. Neural Eng. 6(5), 056003 (2009).

67. H. Cao et al., "An integrated $\mu$ LED optrode for optogenetic stimulation and electrical recording," IEEE Trans. Biomed. Eng. 60(1), 225-229 (2013)

68. P. Anikeeva et al., "Optetrode: a multichannel readout for optogenetic control in freely moving mice," Nat. Neurosci. 15(1), 163-170 (2012).

69. B. Rubehn et al., "A polymer-based neural microimplant for optogenetic applications: design and first in vivo study," Lab Chip 13(4), 579-588 (2013).

70. R. Biran, D. C. Martin, and P. A. Tresco, "Neuronal cell loss accompanies the brain tissue response to chronically implanted silicon microelectrode arrays," Exp. Neurol. 195(1), 115-126 (2005).

71. D. Szarowski et al., "Brain responses to micro-machined silicon devices," Brain Res. 983(1), 23-35 (2003).

72. F. Pisanello et al., "Multipoint-emitting optical fibers for spatially addressable in vivo optogenetics," Neuron 82(6), 1245-1254 (2014).

73. J. G. McCall et al., "Fabrication and application of flexible, multimodal light-emitting devices for wireless optogenetics," Nat. Protoc. 8(12), 2413-2428 (2013)

74. A. J. Yeh et al., "Wirelessly powering miniature implants for optogenetic stimulation," Appl. Phys. Lett. 103(16), 163701 (2013).

75. J. S. Ho et al., "Self-tracking energy transfer for neural stimulation in untethered mice," arXiv preprint arXiv:1503.01493 (2015).

76. J. Silverman, M. A. Suckow, and S. Murthy, The IACUC Handbook, CRC Press (2014). 
77. F. C. Commission, "Guidelines for evaluating the environmental effects of radiofrequency radiation," 1996, https://transition.fcc.gov/Bureaus/ Engineering_Technology/Orders/1996/fcc96326.pdf (29 May 2015).

78. R. Ameli et al., "A wireless and batteryless neural headstage with optical stimulation and electrophysiological recording," in 2013 35th Annual Int. Conf. of the IEEE, pp. 5662-5665 (2013).

79. R. J. Brodd et al., "Batteries, 1977 to 2002," J. Electrochem. Soc. 151(3), K1-K11 (2004).

80. M. Armand and J.-M. Tarascon, "Building better batteries," Nature 451(7179), 652-657 (2008).

81. C. Meng, O. Z. Gall, and P. P. Irazoqui, "A flexible super-capacitive solid-state power supply for miniature implantable medical devices," Biomed. Microdevices 15(6), 973-983 (2013).

82. R. Sharpeshkar, "Ultra Low Power Bioelectronics: Fundamentals, Biomedical Applications, and Bio-Inspired System," Cambridge University Press, Cambridge, UK (2010).

83. M. A. Rossi et al., "A wirelessly controlled implantable LED system for deep brain optogenetic stimulation," Front. Integr. Neurosci. 9, 8 (2015).

84. T.-I. Kim et al., "Injectable, cellular-scale optoelectronics with applications for wireless optogenetics," Science 340(6129), 211-216 (2013).

85. M. M. Elwassif et al., "Bio-heat transfer model of deep brain stimulation-induced temperature changes," J. Neural Eng. 3(4), 306 (2006).

Rajas $\mathbf{P}$. Kale is a $\mathrm{PhD}$ student in neural engineering at Deakin University, Australia, and Mayo Clinic, USA. He received both his bachelor in biomedical engineering and his bachelor of science in neuroscience degrees from the University of Minnesota in 2010. $\mathrm{He}$ is currently developing an optogenetic microdevice to be used on small animals in preclinical models of mood disorder. His research goal is to use novel neurostimulation techniques to expose important biomarkers of neurodegenerative disorders.
Abbas Z. Kouzani received his $\mathrm{PhD}$ in electrical and electronics engineering from Flinders University, Australia. Currently, he is an associate professor with the School of Engineering, Deakin University. He has been involved in over \$2.4 million research grants and has published over 250 refereed papers. His current research interests include medical/biological microdevices, lab-on-a-chip systems, bioinstrumentation, and biosensors and implants. He is the leader of Deakin University's BioMEMS Group.

Ken Walder is chair of metabolic diseases in the School of Medicine, Deakin University and executive director of the Molecular and Medical Research Strategic Research Centre. He has published over 70 peerreviewed research papers primarily in the field of metabolic diseases and has been cited over 2000 times. The focus of his work is the discovery and preclinical development of novel therapeutics for obesity and type 2 diabetes.

Michael Berk is currently an NHMRC senior principal research fellow and is Alfred Deakin chair of psychiatry at Deakin University, where he heads the IMPACT Strategic Research Centre. He is past president of the International Society for Bipolar Disorders and the Australasian Society for Bipolar and Depressive Disorders. He has published over 600 papers predominantly in the discovery and implementation of novel therapies, and risk factors and prevention of psychiatric disorders.

Susannah J. Tye received her PhD from the Departments of Psychology and Biological Sciences, Macquarie University, Australia, in 2008. She is now director of the Translational Neuroscience Laboratory at the Mayo Clinic Depression Center. Her work aims to identify biomarkers of neuropsychiatric disease and treatment response, and translate findings into viable new treatment approaches. Her research has actively contributed to development of animal models of stressinduced depression, mania, and post-traumatic stress disorder. 